\title{
DIETA CETOGÊNICA PARA EPILEPSIA INTRATÁVEL EM CRIANÇAS E ADOLESCENTES: RELATO DE SEIS CASOS
}

\author{
Marcio M. Vasconcelos*, Patricia M. Couto Azevedo, Livia Esteves, \\ Adriana R. Brito, Maria Cecílea D. de Olivaes, Gesmar V. Haddad Herdy \\ Trabalho realizado no Hospital Universitário Antônio Pedro (HUAP) e \\ Universidade Federal Fluminense (UFF), Niterói, Rio de Janeiro, RJ
}

\begin{abstract}
RESUMO - OBJETIVo. Descrever a introdução e o manejo da dieta cetogênica em um grupo de seis crianças e adolescentes com epilepsia refratária.
\end{abstract}

Métodos. Os autores reviram o prontuário médico de cada paciente menor de 15 anos submetido à dieta cetogênica entre abril de 1999 e julho de 2003 e compararam os resultados terapêuticos e efeitos adversos e benéficos com a literatura pertinente.

RESULTADOs. A dieta cetogênica foi introduzida para seis pacientes, com idade mediana de sete anos (faixa: 1,8-12,2). A duração média da aplicação da dieta foi 9,7 meses (faixa: 7 dias-4 anos).
Observou-se uma redução igual ou maior que $50 \%$ da freqüência das crises epilépticas em metade dos casos. As complicaçóes observadas foram leucopenia, constipação, desidratação, priapismo e recorrência das crises epilépticas.

Conclusöes. A dieta cetogênica foi eficaz e segura em três pacientes de uma série de seis casos com epilepsia intratável. A complicação mais comum foi leucopenia.

Unitermos: Epilepsia. Criança. Tratamento. Dieta cetogênica. Corpos cetônicos.

\section{INTRODUÇÃO}

A epilepsia é um dos mais freqüentes e graves distúrbios neurológicos na infância, com incidência aproximada de $1 \%$ da população! Dentre as crianças acometidas, 20\% a 30\% têm crises epilépticas refratárias às drogas antiepilépticas (DAES)2. A consideração de opções não-farmacológicas para o tratamento da epilepsia refratária é oportuna, dadas as evidências de que a frequêencia de crises correlaciona-se fortemente com o prognóstico da epilepsia $^{3}$ e de que o risco de letalidade da epilepsia pediátrica é mais alto nos pacientes com epilepsia intratável/4. Embora a definição de epilepsia intratável seja controversa, comumente recorre-se ao conceito proposto por Schmidt, segundo o qual um paciente deve receber este diagnóstico quando suas crises epilépticas não são controladas por doses máximas toleradas de duas ou três DAES ${ }^{5}$. Existem três opções de tratamento não-farmacológico para o grupo de crianças e adolescentes com epilepsia intratável: cirurgia para epilepsia, estimulação do nervo vago e dieta

*Correspondência:

Av. das Américas, 700 - sala 229 - bloco 6

CEP: 22640-100 - Rio de Janeiro - RJ

Tels: (21) 2132-8080 / 2132-7765

E-mail:mmvascon@centroin.com.br cetogênica (DC), a primeira das quais é inadequada para mais de metade dessas crianças 6 .

A DC foi criada por Wilder ${ }^{7}$, em 1921, na Mayo Clinic, para tratar crianças com epilepsia. Partindo da antiga observação clínica, citada na Bíblia (Mateus 17, | 4-2I), de que o jejum exercia uma ação anticonvulsivante em pacientes epilépticos, Wilder concebeu uma dieta com restrição de carboidratos, taxas minimamente adequadas de proteínas e alto teor de lipídios, a qual mantinha uma produção hepática contínua de corpos cetônicos tanto no estado alimentado quanto no jejum. Em vigência de cetose sangüínea contínua, há uma fase de adaptação do metabolismo cerebral estimada em até 20 dias, depois da qual os neurônios passam a utilizar os corpos cetônicos em lugar da glicose como principal gerador de energia, e o efeito terapêutico é a elevação do limiar convulsivo ${ }^{8}$. Embora se desconheça o mecanismo de ação exato da $D C$, alguns autores demonstraram que os níveis sangüíneos de acetoacetato e $\beta$-hidroxibutirato guardam relação direta, porém não linear, com o grau de proteção contra crises epilépticas, e talvez esta proteção esteja relacionada com um aumento das reservas cerebrais de energia?.

Sabe-se que o potencial cetogênico de uma dieta está em sua capacidade de iniciar e manter a produção dos ácidos acetoacético e $\beta$-hidroxibutírico, o que depende das quantidades e proporções relativas de lipídios, proteínas e carboidratos contidos nos alimentos ingeridos em cada refeição ${ }^{10}$. A glicose anula a produção hepática de corpos cetônicos, via secreção de insulina, porque converte-se facilmente em energia, ao passo que os ácidos graxos são oxidados dentro das mitocôndrias, gerando energia de maneira menos eficiente. Em contrapartida, a oxidação dos lipídios também gera como subprodutos os corpos cetônicos, os quais podem servir de combustível alternativo ${ }^{10}$. Supondo-se uma digestão e absorção completas, a ingestão de $100 \mathrm{~g}$ de carboidratos introduz $100 \mathrm{~g}$ de glicose $\mathrm{e} 0 \mathrm{~g}$ de ácidos graxos no metabolismo; $100 \mathrm{~g}$ de lipídios resultam em $10 \mathrm{~g}$ de glicose (o glicerol é convertido em glicose em uma proporção grama a grama) e $90 \mathrm{~g}$ de ácidos graxos; e $100 \mathrm{~g}$ de proteína fornecem $58 \mathrm{~g}$ de glicose e $46 \mathrm{~g}$ de ácidos graxos (a soma gerada é maior do que 100 porque, após a desaminação, os aminoácidos convertem-se em glicose e ácidos graxos em proporções variáveis) $)^{10,11}$. Estes dados podem ser representados na fórmula:

$$
\frac{K}{A K}=\frac{0,9 L+0,46 P}{C+0, I L+0,58 P}
$$

Na qual $K=$ potencial cetogênico, $A K=$ potencial anticetogênico, $L=$ lipídios, $P=$ proteínas e $\mathrm{C}=$ carboidratos $^{10}$. 
Quando a razão cetogênica, K/AK, de uma determinada dieta ultrapassa I,5, a cetogênese se instala e se expressa clinicamente pelo aparecimento de cetonúria. Em termos práticos, instituir a DC significa manter uma dieta com razão, em gramas, de lipídios para carboidratos e proteínas acima de $3: 1^{10}$. Assim, estabelecem-se a taxa mínima de proteína favorável ao crescimento, em geral I g/kg/dia, e, com base em uma quantidade diária total predeterminada de calorias, a taxa de lipídios. A quantidade de carboidratos é definida pela diferença entre a taxa calórica total e as calorias fornecidas por lipídios e proteínas. Diz-se, por exemplo, que o paciente seguirá uma DC de 4: I, contendo 4 g de lipídios para cada grama de proteínas mais carboidratos.

Em uma tentativa de tornar a DC mais palatável e menos constipante, Huttenlocher utilizou óleo de triglicerídios de cadeia média (TCM) como principal fonte lipídica'2. Como os TCMs são mais cetogênicos que os demais lipídios, é possível oferecer uma maior proporção de proteínas e carboidratos ${ }^{8}$. Contudo, eles encerram a desvantagem de induzir diarréia, a qual tende a ceder com a continuação da dieta'2. Assim, a prática da DC no tratamento da epilepsia infantil abrange duas modalidades básicas: a dieta tradicional ou "clássica" descrita inicialmente, baseada em alimentos com alto teor lipídico como creme de leite, manteiga, maionese, azeite e óleos vegetais, e a dieta à base de TCM.

Devido à descoberta de novas DAES, a partir da década de 1960, aliada às dificuldades em seguir seus princípios rigorosos, a DC foi relativamente esquecida, embora alguns centros, como a Universidade Johns Hopkins ${ }^{13}$, tenham continuado a usá-la. Tornou-se evidente no decorrer do tempo que pelo menos um quinto das crianças epilépticas não respondia favoravelmente a quaisquer das DAES disponíveis ou precisavam de doses excessivas desses medicamentos, com efeitos colaterais intoleráveis. Desse modo, a popularidade da DC cresceu na década de $1990^{\circ}$, e foram publicadas várias séries de casos descrevendo sua eficácial4-16.

Os objetivos deste trabalho são descrever a experiência dos autores com a aplicação da DC clássica a um grupo de seis crianças e adolescentes com epilepsia intratável e enfatizar a eficácia e os elevados benefícios em potencial da DC.

\section{Métodos}

Este é um estudo retrospectivo que avaliou o uso da DC em um grupo de seis crianças e adolescentes com epilepsia previamente intratável, definida aqui pela resposta desfavorável a três drogas antiepilépticas. Os autores reviram o prontuário médico de cada paciente menor de 15 anos submetido à DC, entre abril de 1999 e julho de 2003, com atenção especial às características clínicas da doença subjacente, os exames complementares (eletroencefalograma, exames de imagem, dados laboratoriais), o tipo de crise e, quando possível, a síndrome epiléptica. Estudou-se o número de DAE previamente usadas. Analisaramse os seguintes aspectos da DC: eficácia (considerou-se como critério de eficácia uma redução igual ou maior que $50 \%$ na freqüência de crises epilépticas), efeitos benéficos associados (p. ex., sobre o humor ou a cognição), tolerância, dificuldade de aplicação, alterações laboratoriais e complicações. A base de comparação da freqüência de crises eram as últimas quatro semanas antes da DC, registradas em diário pelos pais. À internação para introdução da DC, cada crise epiléptica era registrada pela equipe médica e de enfermagem e, após a alta hospitalar, os pais foram orientados a manter um registro sistemático da hora, duração e características de cada crise convulsiva.

\section{Protocolo da dieta cetogênica}

Os autores seguiram a DC clássica, conforme descrita por Wilder ${ }^{7}$ e divulgada por Freeman et al. ${ }^{17,18}$. Os critérios de inclusão no estudo foram idade do paciente entre I e 15 anos, uso prévio de pelo menos três DAES apropriadas em doses máximas, ausência de acidose metabólica ou algum outro distúrbio metabólico definido, capacidade psicossocial da família em aplicar a dieta e ausência de doenças intercorrentes. Cada paciente foi submetido a uma anamnese completa, exames físico e neurológico minuciosos e entrevista psicossocial dos responsáveis. Os pais e todos os adultos que conviviam com cada paciente foram instruídos durante no mínimo duas consultas acerca dos benefícios e riscos em potencial da DC, dificuldades na implantação da dieta e erros freqüentes na sua execução, e tiveram de assinar um consentimento informado para que o paciente fosse submetido à DC. Não houve autorização prévia da Comissão de Ética em Pesquisa da instituição dos autores porque os seis pacientes não foram assistidos dentro de um protocolo formal de estudo. Antes da internação, realizaram-se exames laboratoriais para excluir infecções ou outras intercorrências, e foi solicitado aos pais observar a criança atentamente com o objetivo de definir a frequêencia de crises epilépticas pré-DC. Os exames preliminares foram hemograma completo, gasometria arterial, perfil bioquímico sangüíneo (incluindo o sódio, potássio e cloreto; cálcio, fosfato, magnésio e fosfatase alcalina; aspartato-aminotransferase, alanina-aminotransferase e bilirrubina total; glicemia; uréia e creatinina), exame de urina e urocultura, exame parasitológico de fezes, proteínas séricas e lipidograma.

A introdução da DC exigia uma internação de no mínimo cinco dias, realizada após a verificação dos resultados dos exames de triagem. Os autores adotaram o recurso de manter o paciente em jejum até o aparecimento de cetose plena (detectada através de cetonúria no grau 4+). Durante o jejum, o paciente foi mantido com hidratação intravenosa sem glicose em dois terços da taxa hídrica plena e monitorado com aferição dos sinais vitais e medição da glicemia capilar e cetonúria a cada seis horas. Uma vez ao dia, enviavam-se ao laboratório amostras para a glicemia, eletrólitos, ácido úrico, uréia e creatinina, aminotransferases hepáticas e exame de urina. Uma vez instalada a cetose plena, a DC era iniciada. As refeições foram introduzidas em volumes progressivos, I/3 do volume no primeiro dia, 2/3 no segundo dia e volume total no terceiro dia. Para facilitar a preparação da dieta no hospital, os autores optaram por fornecer apenas a gemada (contendo creme de leite com teor de lipídios de 35\%, ovo e triglicerídios de cadeia média) nas três refeições diárias ${ }^{17}$. Durante a internação, a equipe médica e de enfermagem observava e registrava a hora e duração de cada crise clínica; os pais foram instruídos na preparação rigorosa das refeições no lar e no manejo de uma balança eletrônica com escala em gramas; neste estudo, utilizaram-se balanças eletrônicas portáteis da marca Tanita, 
Tabela I - Resumo dos seis pacientes submetidos à dieta cetogênica

\begin{tabular}{|c|c|c|c|c|c|c|c|c|}
\hline & \multirow[b]{2}{*}{ Sexo } & \multirow[b]{2}{*}{$\begin{array}{l}\text { Idade no } \\
\text { início da } \\
\text { DC (anos) }\end{array}$} & \multirow[b]{2}{*}{$\begin{array}{c}\text { Tipo de crise } \\
\text { ou síndrome } \\
\text { epiléptica' }\end{array}$} & \multirow[b]{2}{*}{$\begin{array}{c}\text { Quadro } \\
\text { neurológico }\end{array}$} & \multirow[b]{2}{*}{ Eletroencefalograma ${ }^{3}$} & \multirow[b]{2}{*}{$\begin{array}{l}\text { Exames de } \\
\text { imagem }^{4}\end{array}$} & \multicolumn{2}{|c|}{ Drogras antiepilépticas $^{5}$} \\
\hline & & & & & & & Prévias & $\begin{array}{c}\text { No início } \\
\text { da DC }\end{array}$ \\
\hline CASOI & Fem & 4,5 & $\begin{array}{c}\text { CPC com } \\
\text { generalização } \\
\text { secundária }\end{array}$ & AGD & $\begin{array}{c}\text { Pontas-ondas } \\
\text { lentas bilaterais } \\
\text { e independentes } \\
(E>D) ; \\
\text { atividade de } \\
\text { base suprimida } \\
\text { à E. }\end{array}$ & $\begin{array}{l}\text { RME aos } 3 \text { anos } \\
\text { normal; RME } \\
\text { aos 4,5 anos: } \\
\text { atrofiacerebral }\end{array}$ & $\begin{array}{l}\text { CBZ, CLB, } \\
\text { DZP, GBP, } \\
\text { LTG, PB, } \\
\text { PHT, CGB, } \\
\text { VPA }\end{array}$ & $P B$ \\
\hline$\overline{\mathrm{CASO} 2}$ & Masc & 1,8 & $\begin{array}{l}\text { Espasmos } \\
\text { infantis, } \\
\text { miocloniase } \\
\text { CPC }\end{array}$ & $\begin{array}{l}\text { Esclerose } \\
\text { tuberosa }\end{array}$ & Hipsarritmia & $\begin{array}{c}\text { TCC: } \\
\text { calcificações } \\
\text { subependimárias }\end{array}$ & $\begin{array}{l}\text { NZP, PB, } \\
\text { VPA }\end{array}$ & VPA \\
\hline$\overline{\mathrm{CASO} 3}$ & Masc & 7,9 & CPC, TCG & AGD & $\begin{array}{l}\text { Hipsarritmia } \\
\text { modificada }\end{array}$ & TCCnormal & $\begin{array}{c}\text { DZP, OCBZ, } \\
\text { PB }\end{array}$ & OCBZ, PB \\
\hline$\overline{\mathrm{CASO}} 4$ & Masc & 6,1 & $\begin{array}{c}\text { TCG, } \\
\text { mioclonias, } \\
\text { ausência atíicica }\end{array}$ & $A G D$ & $\begin{array}{l}\text { Pontas-ondas } \\
\text { lentas bifrontais }\end{array}$ & RME normal & $\begin{array}{c}\text { CBZ, CLB, } \\
\text { DZP, LTG, PB, } \\
\text { TPM, VPA }\end{array}$ & CBZ, PB \\
\hline CASO 5 & Masc & 12,2 & $\begin{array}{l}\text { TCG, crises } \\
\text { atônicase } \\
\text { tônicas }\end{array}$ & AGD & $\begin{array}{c}\text { Traçado } \\
\text { compatível com } \\
\text { SLG }\end{array}$ & $\begin{array}{l}\text { TCCe RME } \\
\text { normais. }\end{array}$ & $\begin{array}{l}\text { CBZ, CNZ, } \\
\text { PB, VPA }\end{array}$ & $\begin{array}{c}C B Z, \\
C N Z, P B\end{array}$ \\
\hline CASO 6 & Masc & 11,5 & $\begin{array}{l}\text { CPC, às vezes } \\
\text { generalização } \\
\text { secundária }\end{array}$ & $\begin{array}{l}\text { Hipomelanose } \\
\text { de lto }\end{array}$ & $\begin{array}{l}\text { Pontas-ondas } \\
\text { lentas à E }\end{array}$ & RME normal & $\begin{array}{l}\text { CBZ, DZP, } \\
\text { LTG, OCBZ, } \\
\text { PHT, TPM, } \\
\text { VPA }\end{array}$ & CBZ, TPM \\
\hline
\end{tabular}

*Abreviaturas:

' CPC; crises parciais complexas; TCG = convulsão tônico-clônica generalizada.

${ }^{2} A G D=$ atraso global do desenvolvimento

${ }^{3} \mathrm{D}=$ direita; $E$ = esquerda; $S L G$ = síndrome de Lennox-Gastaut.

${ }^{4} \mathrm{RME}=$ ressonância magnética do encéfalo; $T C C=$ tomografia computadorizada de crânio.

${ }^{5}$ Por convenção, usam-se as abreviaturas internacionais ${ }^{28}: \mathrm{CBZ}=$ carbamazepina; $C L B=$ clobazam; CNZ = clonazepam; DZP = diazepam; GBP = gabapentina; $L T G=$ lamotrigina; $N Z P=$ nitrazepam;

$O C B Z=$ oxcarbazepina; $P B=$ fenobarbital; $P H T=$ fenitoína; $T P M=$ topiramato; $V G B=$ vigabatrina; $V P A=$ ácido valpróico.

modelo ||40. Após a alta hospitalar, o paciente passava a receber suplementos de cálcio, ferro e um polivitamínico. 0 acompanhamento ambulatorial incluiu revisões semanais durante as primeiras quatro semanas, consultas mensais nos três meses seguintes e, então, consultas bimestrais regulares. Além do registro diário das crises, os pais foram incentivados a verificar a cetonúria matinal diariamente com tira reagente específica ao longo de toda a duração da DC.

A DC foi calculada usando-se o software Keto Diet Meal Planner, distribuído gratuitamente pelo Lucile Packard Children's Hospital da Universidade de Stanford, nos Estados Unidos (porém, foi preciso adaptar os valores nutricionais dos alimentos aos produtos nacionais). Adotaram-se os seguintes parâmetros para cálculo das refeições: razão cetogênica inicial (proporção entre gramas de lipídios e gramas de proteínas + carboidratos) de 4: I, taxa diária de proteína de I g por kg de peso corporal, taxa calórica calculada em aproximadamente dois terços das necessidades de manutenção tendo como base o peso ideal, número fixo inicial de três refeições diárias e taxa hídrica um pouco abaixo das necessidades de manutenção e limitadas a I ml por quilocaloria ao dia. À alta hospitalar, cada criança recebia um cardápio com cinco ou seis refeições rigorosamente calculadas com proporções nutricionais idênticas, que podiam ser oferecidas em qualquer uma das três refeições diárias.

\section{Resultados}

No total, seis pacientes (cinco meninos e uma menina) foram submetidos à DC. A idade mediana no início da dieta foi de sete anos (faixa: 1,8 a 12,2 anos). A duração média da aplicação da dieta foi 9,7 meses (faixa: 7 dias4 anos). Observou-se uma redução igual ou maior que $50 \%$ da freqüência das crises epilépticas em três dos seis casos estudados.

A Tabela I resume as características clínicas dos seis pacientes. A Tabela 2 mostra as complicações, o efeito da DC sobre as crises, a cognição dos pacientes e os resultados laboratoriais observados.

A execução da DC foi dificultada por intercorrências em dois casos. No sexto dia de internação do caso 2, a avó visitou a criança e, assustada com sua astenia, decidiu alimentá-la 


\section{Tabela 2 - Parâmetros e resultados da dieta cetogênica em seis pacientes com epilepsia intratável}

\begin{tabular}{|c|c|c|c|c|c|c|c|}
\hline & $\begin{array}{c}\text { Razão } \\
\text { cetogênica }\end{array}$ & $\begin{array}{c}\text { Taxa calórica } \\
\text { diária' }\end{array}$ & Duração & Complicações & $\begin{array}{l}\text { Efeito sobre } \\
\text { as crises }\end{array}$ & $\begin{array}{c}\text { Efeito sobre a } \\
\text { cognição }\end{array}$ & Laboratório $^{2}$ \\
\hline CASO I & 4:1, depois 5:1 & 60 & $\overline{4 \text { anos; em uso }}$ & $\begin{array}{l}\text { Leucopenia, } \\
\text { constipação }\end{array}$ & $\overline{\text { Redução }>50 \%}$ & $\begin{array}{c}\text { Deambulação } \\
\text { independente, } \\
\text { voltou a falar }\end{array}$ & $\begin{array}{c}\text { G42, AU9,8 } \\
\text { pH 7,28, } \\
\text { Col 192, TG65,Leuc 3,6 }\end{array}$ \\
\hline$\overline{\mathrm{CASO}} 2$ & $4: 1$ & 75 & 7 dias & $\begin{array}{l}\text { Desidratação e } \\
\text { letargia }\end{array}$ & $\begin{array}{c}\text { Sem crises até } \\
\text { a suspensão }\end{array}$ & - & $\begin{array}{c}\text { G38, AU9,5, } \\
\text { pH 7,35 }\end{array}$ \\
\hline$\overline{\mathrm{CASO} 3}$ & $4: 1$ & 60 & $\begin{array}{c}25 \text { meses; em } \\
\text { uso }\end{array}$ & $\begin{array}{l}\text { Baixo ganho } \\
\text { ponderal, } \\
\text { leucopenia }\end{array}$ & Redução > 50\% & $\begin{array}{c}\text { Melhora da fala, } \\
\text { deambulação } \\
\text { independente }\end{array}$ & $\begin{array}{c}\mathrm{G} 61, \mathrm{pH} 7,31, \\
\text { Col 220, Tg 139, } \\
\text { Leuc 3,4 }\end{array}$ \\
\hline$\overline{\mathrm{CASO}} 4$ & $4: 1$ & 68 & 52 dias & $\begin{array}{c}\text { Recorrênciadas } \\
\text { crises }\end{array}$ & $\begin{array}{l}\text { Redução }>50 \% \\
\text { no ińício; depois, } \\
\text { crises de ausência } \\
\text { persistentes }\end{array}$ & $\begin{array}{c}\text { Melhora } \\
\text { transitória da } \\
\text { fala }\end{array}$ & $\begin{array}{c}\text { G 48, AU8,3, } \\
\text { pH 7,29 }\end{array}$ \\
\hline$\overline{\mathrm{CASO}} 5$ & $4,25: 1$ & 52 & $\begin{array}{c}18 \text { meses; em } \\
\text { uso }\end{array}$ & $\begin{array}{l}\text { Leucopenia, } \\
\text { constipação }\end{array}$ & $\begin{array}{c}\text { Sem crises há } 13 \\
\text { meses }\end{array}$ & $\begin{array}{l}\text { Melhora da fala, } \\
\text { deambulação } \\
\text { independente, } \\
\text { menos sialorréia }\end{array}$ & $\begin{array}{c}\text { G 45, pH7,21, } \\
\text { Col 262, Tg 79, } \\
\text { Leuc } 2,69\end{array}$ \\
\hline$\overline{\text { CASO } 6}$ & $\begin{array}{c}3,5: 1 \text {, depois } \\
3,75: 1\end{array}$ & 42 & 51 dias & $\begin{array}{l}\text { Perda de } 20 \% \text { do } \\
\text { peso, priapismo }\end{array}$ & Piora das crises & Melhoraglobal & $\begin{array}{c}\text { G52, AU 8,9, } \\
\text { pH 7,31, } \\
\text { Col 20I, Tg99 }\end{array}$ \\
\hline
\end{tabular}

Em quilocalorias por quilograma de peso corporal por dia.

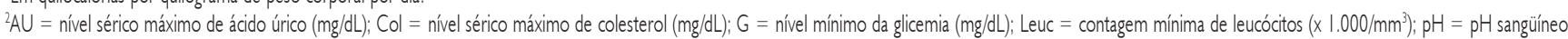
arterial mínimo; $\mathrm{Tg}$ = nível sérico máximo de triglicerídios $(\mathrm{mg} / \mathrm{dL})$.

com suco de laranja. A cetose foi prontamente interrompida pelo aporte de carboidrato. Este incidente levou à suspensão da dieta, a pedido da mãe do paciente, apesar da excelente resposta terapêutica inicial. $O$ caso 6 apresentou dois episódios de recorrência das crises epilépticas ao longo de 13 meses: o primeiro quando ingeriu uma única batata frita e apresentou uma crise clônica breve, e o segundo no dia do aniversário da irmã, quando houve quebra da cetose e ele teve duas crises epilépticas noturnas - provavelmente por ter transgredido a dieta durante a festa.

\section{Discussão}

Neste estudo, três em seis crianças com epilepsia submetidas à DC mostraram uma redução persistente maior ou igual a $50 \%$ da freqüência de crises epilépticas. Embora a baixa potência estatística de uma série de apenas seis casos não permita definir a eficácia do tratamento, o resultado foi comparável à taxa de eficácia de $54 \%$ no estudo multicêntrico prospectivo envolvendo $5 \mathrm{I}$ crianças de Vining et al. ${ }^{16} \mathrm{e}$ um pouco inferior à taxa de $62 \%$ descrita em um estudo retrospectivo de 58 crianças por Kinsman et al. ${ }^{14}$.
Antes de oferecer a opção terapêutica da DC à família de uma criança com epilepsia supostamente intratável, é preciso considerar que o fracasso do tratamento farmacológico pode decorrer de diversos outros fatores, como erro de diagnóstico, dose insuficiente ou horário impróprio da medicação, baixa adesão à terapia, classificação errônea do tipo de crise epiléptica, ou a presença de uma doença progressiva do sistema nervoso central ${ }^{3}$. Nos seis pacientes incluídos neste estudo, o diagnóstico de epilepsia intratável foi escrupulosamente revisto e confirmado. Todos os pacientes haviam recebido no mínimo três DAES em doses máximas (Tabela I).

O uso do relato pelos pais da freqüência de crises epilépticas para aferir a resposta à DC não é o método ideal para avaliar a eficácia de uma terapia antiepiléptica, mas os três pacientes que responderam à dieta exibiram melhora acentuada da função global (p. ex., de ambulação independente) associada à redução ou resolução das crises. Embora imperfeito, o registro diário pelos pais da freqüência de crises também foi utilizado em estudos prévios ${ }^{14-16}$, e parece ser o método mais prático e exeqüível em estudos de acompanhamento a longo prazo sobre epilepsia.
Alguns autores afirmam que a DC não é tão utilizada porque ela é pouco palatável ${ }^{14}$, mas a prática da dieta demonstra que sua aceitação pode ser alta. Desde que percebam uma melhora significativa no estado clínico de seus filhos, pais e familiares costumam tolerar a rigidez na aplicação da DC para manter a cetose sangüínea contínua ${ }^{18}$. Porém, os incidentes descritos no caso 2, quando a avó da criança infringiu a DC, e no caso 6 , quando a ingestão de uma única batata frita desencadeou crise epiléptica, devem servir de alerta para a necessidade de uma explicação detalhada da introdução e da manutenção da DC a todos os familiares e responsáveis que conviverão com - paciente, enfatizando os percalços e os efeitos colaterais possíveis.

Com o aumento no interesse pela DC a partir de 19948, deu-se maior atenção à toxicidade e aos efeitos colaterais da dieta ${ }^{19-}$ ${ }^{23}$. Na presente série de casos, todas as três crianças tratadas com a DC por período superior a dois meses apresentaram leucopenia (contagem de leucócitos < 4.500 células/ $\mathrm{mm}^{3}$ ), uma complicação ainda não citada na literatura consultada pelos autores. Os casos I, 3 e 5 apresentaram contagens totais de leucócitos mínimas, respectivamente, de 
$3.600,3.400$ e 2.690 células $/ \mathrm{mm}^{3}$, após períodos de tempo em uso da DC de 5 a 22 meses. A leucopenia não foi acompanhada de complicações infecciosas e, nos três casos, remitiu espontaneamente. Woody et al..$^{19}$ descreveram uma deficiência da função dos neutrófilos em nove crianças de um a nove anos de idade tratadas com a DC, mas não mencionaram qualquer alteração quantitativa dos leucócitos. A ocorrência de priapismo durante a primeira semana de uso da DC no caso 6 também não fôra mencionada na literatura, mas não é possível definir se este adveio ou não da introdução da DC. A acidose metabólica foi leve a moderada e transitória no início da DC nos seis pacientes estudados, mas eventualmente é grave o suficiente para motivar a suspensão da $D C^{18}$.

Outros eventos adversos em potencial da DC incluem alteração do estado mental (até mesmo coma), hiperlipidemia, hiperuricemia, hipocalcemia, hipoglicemia, desidratação, constipação ou diarréía, recusa alimentar, desmineralização óssea, litíase renal e infecções recorrentes ${ }^{21}$. Em um relato de cinco crianças que apresentaram complicações sérias e ameaçadoras à vida durante o tratamento com a DC, como perda ponderal, disfunção hepática, hipoproteinemia e acidose tubular renal, Ballaban-Gil et al. enfatizaram a necessidade de supervisão estreita quando a DC é associada ao uso de ácido valpróico, o qual pode inibir a fosforilação oxidativa ${ }^{21}$. Um relato recente mencionou a possibilidade de complicações cardíacas em crianças submetidas à DC22; houve um prolongamento do intervalo QTc em três de 20 crianças submetidas à DC, porém apenas uma criança necessitou que a dieta fosse suspensa.

A introdução da DC clássica preconiza um período de jejum inicial para acelerar a cetogênese e obter o efeito terapêutico desejado. Como este período de jejum é exigente do ponto de vista metabólico e encerra um risco mais alto de descompensação clínica ( $p$. ex., o caso 2), a recomendação recente de iniciar a DC sem jejum é promissora; Wirrell et al. ${ }^{24}$ descreveram 14 crianças submetidas à DC sem jejum inicial, com aumento gradual da taxa cetogênica da dieta de 1:I até 3:1 ou 4:1 ao longo de três a quatro dias. $O$ tempo médio até a obtenção de cetose plena foi de 58 horas (faixa: 40 a 84 horas). A cetose foi alcançada por 13 das 14 crianças.
A aplicação da DC também foi sugerida para o manejo nutricional de crianças com determinados distúrbios metabólicos, como a deficiência da proteína transportadora de glicose (doença de DeVivo), deficiência de piruvato-desidrogenase e defeitos da glicólise cerebral ${ }^{25}$. Por outro lado, deve-se realizar uma triagem metabólica antes de instituir a DC, pois determinados distúrbios metabólicos podem ser agravados pela dieta, como a deficiência de piruvato-carboxilase, a porfiria, a deficiência de carnitina, os distúrbios mitocondriais e os defeitos da oxidação dos ácidos graxos ${ }^{26}$.

\section{Conclusão}

Considerando-se a redução dos custos da assistência médica de crianças com epilepsia intratável quando a DC é bem-sucedida ${ }^{27}$ e as taxas de eficácia da DC de até $62 \%$ citadas na literatura ${ }^{14,16}$, os autores do presente estudo acreditam que sempre que possível a DC deve ser instituída como uma prova terapêutica às crianças com epilepsia intratável, mesmo antes de se contemplar uma opção terapêutica mais definitiva e invasiva como a cirurgia para epilepsia.

\section{Agradecimento}

Os autores desejam agradecer à nutricionista Ana Lúcia Pires Augusto, Profa. Assistente do Departamento de Nutrição e Dietética da Universidade Federal Fluminense, por sua participação competente e prestimosa na implantação da dieta cetogênica no Hospital Universitário Antônio Pedro.

\section{Conflito de interesse: não há.}

\section{SUMMARY}

\section{KETOGENIC DIET FOR INTRACTABLE EPILEPSY IN CHILDREN AND ADOLESCENTS: REPORT OF SIX CASES}

BACKGROUND. Thisstudyaims to reporton use of the ketogenic diet in a group of six children and adolescents with intractable epilepsy.

MetHODS. Authors reviewed the medical records of every patient under 15 years of age who received the ketogenic diet between April 1999 and July 2003. A comparison is made between treatment results, adverse events and beneficial effects with the pertinent medical literature.
RESULTS. The ketogenic dietwas administered to six patients, whose median age was 7.0 years (range, 1.8-12.2). Average duration of diet application was of 9.7 months (range, 7 days- 4 years). A reduction equal to orgreater than $50 \%$ in seizure frequency was observed in half of the cases. Complications included neutropenia, constipation, dehydration, priapism, and seizure recurrence.

Conclusions. The ketogenic dietwaseffective and safe in three out of six patients with intractable epilepsy. Neutropenia was the most common complication. [Rev Assoc Med Bras 2004; 50(4): 380-5]

Key words: Epilepsy. Child. Therapy. Ketogenic diet. Ketones.

\section{ReFERÊNCIAS}

I. Yacubian EMT. Tratamento da epilepsia na infância. J Pediatr (Rio J) 2002; 78:S 19-S27.

2. Costa JC. Tratamento cirúrgico das epilepsias na criança. J Pediatr (Rio J) 2002; 78:\$28-539

3. Aicardi J. General aspects of prognosis. In: Aicardi J. Epilepsy in children. $2^{\text {nd }}$ ed. Philadelphia: Lippincott-Raven; 1996. p.381-93.

4. Shinnar S, Pellock JM. Update on the epidemiology and prognosis of pediatric epilepsy. J Child Neurol 2002; 17(Suppl I): S4-17.

5. Schmidt D. Diagnostic and therapeutic management of intractable epilepsy. In: Schmidt, D, Morselli, PL. Intractable epilepsy: experimental and clinical aspects. New York: Raven Press, 1986. p.237-57.

6. Sheth RD, Stafstrom CE. Intractable pediatric epilepsy: vagal nerve stimulation and the ketogenic diet. Neurol Clin North Am 2002; 20: $1183-94$.

7. Wilder RM. The effect of ketonemia on the course of epilepsy. Bull Mayo Clin 1921; 2:307-8.

8. Swink TD, Vining EPG, Freeman JM. The ketogenic diet: 1997. Adv Pediatr 1997; 44:297-329.

9. Vining EPG. The ketogenic diet. Adv Exp Med Biol 2002; 497:225-3 I.

10. Withrow CD. Antiepileptic Drugs. The Ketogenic diet: mechanism of anticonvulsant action. In Glaser GH, Penry JK, Woodbury DM, editors. Antiepileptic drugs: mechanisms of action. New York: Raven Press; 1980. p.635-42

II. Woodyatt RT. Objects and method of diet adjustment in diabetes. Arch Intern Med 1921; 28: |25-4|.

12. Huttenlocher PR. Ketonemia and seizures: metabolic and anticonvulsant effects of two ketogenic diets in childhood epilepsy. Pediatr Res 1976; 10:536-540. 
13. Livingston S, Pauli LL, Pruce I. Ketogenic diet in the treatment of childhood epilepsy. Dev Med Child Neurol 1977; 19:833-4.

14. Kinsman SL, Vining EPG, Quaskey SA, Mellits $D$, Freeman JM. Efficacy of the ketogenic diet for intractable seizure disorders: review of 58 cases. Epilepsia 1992; 33:1132-6.

15. Vasconcelos MM, Packer RJ, Weinstein S, Kolodgie M, Goodwin M, Conry JA, et al. Efficacy and toxicity of the ketogenic diet in children with intractable epilepsy. Ann Neurol 1995; 3:505. [abstract].

16. Vining EPG, Freeman JM, Ballaban-Gil K, Camfield CS, Camfield PR, Holmes GL, et al. A multicenter study of the efficacy of the ketogenic diet. Arch Neurol 1998; 55: I 433-7.

17. Freeman JM, Freeman JB, Kelly MT. The ketogenic diet: a treatment for epilepsy. $3^{\mathrm{a}} \mathrm{ed}$. New York: Demos Medical Publishing; 2000.

18. Freeman J, Vining EPG, Pillas DJ, Pyzik PL, Casey JC, Kelly MT. The efficacy of the ketogenic diet-1998: a prospective evaluation of intervention in 150 children. Pediatrics 1998; 102:1358-63.
19. Woody RC, Steele RW, Knapple WL, Pilkington NS. Impaired neutrophil function in children with seizures treated with the ketogenic diet. J Pediatr 1989; I I 5:427-30.

20. Herzberg GZ, Fivush BA, Kinsman SL, Gearhart JP. Urolithiasis associated with the ketogenic diet. J Pediatr 1990; I 17:743-5

21. Ballaban-Gil K, Callahan C, O'Dell C, Pappo M, Moshé S, Shinnar S. Complications of the ketogenic diet. Epilepsia 1998; 39:744-8.

22. Best TH, Franz DN, Gilbert DL, Nelson, DP, Epstein MR. Cardiac complications in pediatric patients on the ketogenic diet. Neurology 2000; 54:2328-30

23. Ríos VG, Panico LR, Demartini MG, Carniello MA. Complicaciones en el tratamiento de la epilepsia con dieta cetogénica. Rev Neurol 2001; 33:909-15.

24. Wirrell EC, Darwish HZ, Williams-Dyjur C, Blackman $M$, Lange $V$. Is a fast necessary when initiating the ketogenic diet? J Child Neurol 2002:17:179-82
25. Nordli D. The ketogenic diet: uses and abuses. Neurology 2002;58(Suppl 7): S2I-S4

26. Kossoff EH, Pyzik PL, McGrogan JR, Vining EPG, Freeman JM. Efficacy of the ketogenic diet for infantile spasms. Pediatrics 2002; 109:780-3.

27. Mandel A, Ballew M, Pina-Garza JE, Stalmasek V, Clemens LH. Medical costs are reduced when children with intractable epilepsy are successfully treated with the ketogenic diet. J Am Diet Assoc 2002; 102:396-8.

28. International League Against Epilepsy. Official names of antiepileptic drugs. Epilepsia 1993; 34:II51.

Artigo recebido: 20/10/03 Aceito para publicação: 02/04/04

\section{www.ramb.org.br}

A Ramb disponibiliza agora um novo serviço para os nossos colaboradores:

0 envio de artigos pela internet. Através do nosso publicador, que agiliza o fluxo de submissão com mais rapidez e versatilidade, você poderá enviar seu artigo para publicação na Ramb. Experimente: é muito mais cômodo, muito mais rápido e você ainda poderá acompanhar on line o curso de sua apreciação.

Acesse www.ramb.org.br 